\title{
Preemptive analgesic effect of magnesium sulfate on postoperative pain in patients undergoing lumbar fusion surgery
}

\author{
Abasali Delavari*, Marzieh Lak*, Hasan Araghizadeh*, and Babak Salatini*
}

\section{ABSTRACT}

\section{BACKGROUND}

Reducing postoperative pain can improve patient satisfaction and hospital cost. Intravenous magnesium sulfate is one of the proposed drugs for preemptive analgesia. The study aimed to evaluate the effect of magnesium sulfate on postoperative pain in patients undergoing lumbar fusion surgery.

\section{METHODS}

A double-blind randomized clinical trial was conducted on candidates for vertebral fusion surgery with American Society of Anesthesiologists (ASA) class I-II. One hundred and two patients were randomized into the magnesium sulfate group and control group. The magnesium sulfate group received magnesium sulfate at a dose of $50 \mathrm{mg} / \mathrm{kg}$ in $20 \mathrm{~mL}$ volume and infused during 15-30 minutes pre-operation, while the control group received $20 \mathrm{~mL}$ normal saline. The severity of the pain was assessed by the Visual Analogue Scale (VAS) at 2, 4, 6, 12 and 24 hours after patients entering the post-anesthetic care unit. After the operation, the patients' relaxation rate was assessed based on Ramsay sedation score (RSS).

\section{RESULTS}

There was no significant difference at 2, 4, 6, 12 and 24 hours post-operation between the two groups in the mean severity of pain $(p>0.05)$. There was no significant difference at 2, 4, 6, 12 and 24 hours post-operation between the two groups in the mean of pethidine consumption. There was no significant difference in the relaxation of the patients according to the RSS criteria in the magnesium sulfate and control groups $(\mathrm{p}=0.162)$.

\section{CONCLUSION}

Pre-operative administration of magnesium sulfate does not affect reduction in postoperative pain and opioid consumption of patients undergoing lumbar fusion surgery.

Keywords: Pain, magnesium sulfate, vertebral fusion surgery
*Trauma Research Center, Baqiyatallah University of Medical Sciences, Tehran, Iran

Correspondence:

Marzieh Lak

Anesthesiologist, Trauma Research Center, Baqiyatallah University of Medical Sciences, Tehran, Iran Tel: +989125262585 E-mail: marziehlak@yahoo.com ORCID ID: 0000-0003-3498-4653

Date of first submission, October 24 , 2018

Date of final revised submission, October 9, 2019

Date of acceptance, October 14, 2019

This open access article is distributed under a Creative Commons AttributionNon Commercial-Share Alike 4.0 International License

Cite this article as: Delavari, Lak, Araghizadeh, et al. Preemptive analgesic effect of magnesium sulfate on postoperative pain in patients undergoing lumbar fusion surgery. Univ Med 2019;38:156-63. doi: 10.18051/ UnivMed.2019.v38.156-163 


\section{INTRODUCTION}

Postoperative pain is one of the most common complications of all surgical procedures. There are currently various methods to control postoperative pain, ${ }^{(1)}$ one of which is preemptive analgesia. In this method prior to surgery and tissue damage, the medicine is administered to the patients. ${ }^{(2)}$ These medications through controlling the pain pathways and preventing the release of pain mediators can better control postoperative pain. ${ }^{(3)}$ Preemptive analgesia is the strategy of preventing the establishment of pain signals and is designed to prevent central sensitization. To reduce acute pain after tissue damage and preventing postoperative pain and its spread is the main purpose of preemptive analgesia. ${ }^{(4)}$ Intravenous magnesium sulfate administration is one of the most effective methods of preemptive analgesia. ${ }^{(5)}$

The second most important intracellular ion after potassium is magnesium that is effective in several physiological functions and enzymatic activities. ${ }^{(6,7)}$ Other magnesium tasks are protein synthesis, vasomotor system adjustment, and neurotransmitter. ${ }^{(6,8)}$ Magnesium also plays a role as a non-competitive $\mathrm{N}$-methyl $\mathrm{D}$-aspartate (NDMA) glutamate receptor antagonist that is involved in the acute pain process as well as postoperative pain. The mechanism of the analgesic effect of magnesium sulfate is by interfering with calcium channels and NMDA receptors, as well as reducing the release of catecholamine. Magnesium sulfate by increasing the pain threshold also helps prevent pain. ${ }^{(9-13)}$ Since 1990 , the effect of magnesium sulfate has been studied on postoperative analgesia and decreased postoperative opioid consumption. ${ }^{(6,14-16)}$

Several studies have reported contradictory results of the effect of magnesium sulfate on postoperative pain relief. Some of these studies suggest that pre-operative magnesium sulfate significantly reduced postoperative pain. ${ }^{(17,18)}$ However, some other studies do not support that. ${ }^{(16,19)}$ The aim of the present study was to evaluate the effect of magnesium sulfate on postoperative pain in patients undergoing lumbar fusion surgery.

\section{METHODS}

\section{Research design}

A double-blind randomized clinical trial was conducted in Baqiyatallah Hospital between February 2018 and January 2019.

\section{Research subjects}

All patients who were candidates for vertebral fusion surgery with American Society of Anesthesiologists (ASA) class I-II and aged between 20 to 70 years were included in the study. By a computer-generated randomization table, patients were divided into two groups: magnesium sulfate (MS) group $(\mathrm{n}=51)$ and control group (normal saline group) $(\mathrm{n}=51)$.

The exclusion criteria included a history of previous spinal surgery, major systemic disorders in vital organs such as liver, kidney and heart, magnesium sulfate susceptibility, use of calcium blockers, pregnant women, neurological disorders, opiate addiction patients, a history of psychological illness or history of taking psychiatric drugs, BMI less than 30. Patients who consumed a non-protocol drug during the study were also excluded.

\section{Sample size determination}

The sample size determination was according to a moderate effect size of 0.5 , a statistical power of $80 \%$, and a confidence coefficient of $95 \%$, and yielded 51 cases per group. For this sample size determination, Gpower-3.1.7 software and its sample size formula for comparing two means between two groups were used.

\section{Interventions}

A special code was assigned to each syringe using a random number table. The syringes containing magnesium sulfate and 
control were completely uniform in terms of color and specification, and the patients and anesthesiologist who injected anesthetic drugs were not aware of the type of syringe content. One of the authors was aware of the number of cases and grouping, and also the syringe content and this author delivered syringes to the anesthesiologist.

The magnesium sulfate group received magnesium sulfate at a dose of $50 \mathrm{mg} / \mathrm{kg}$ in $20 \mathrm{~mL}$ volume and infused during 15-30 minutes preoperation, while the control group received $20 \mathrm{~mL}$ normal saline. The main outcomes were dose of propofol, remifentanil, and pethidine, VAS score, and Ramsay sedation score. Regular monitoring was performed every five minutes on blood pressure, pulse oximetry and electrocardiogram (ECG) during the injection of magnesium sulfate, perioperatively and during recovery.

During syringe injection, patients were monitored for adverse drug reactions every five minutes. In the presence of complications of magnesium sulfate, such as dizziness, drowsiness, hypotension or flushing, the rate of drug injection was reduced. In case of hypotension when mean arterial pressure (MAP) $<65 \mathrm{~mm} \mathrm{Hg}$ or there was a reduction in systolic blood pressure of $30 \%$ relative to the initial blood pressure, ephedrine at a dose of 5 to $10 \mathrm{mg}$ was used.

After completion of magnesium sulfate administration, the patients were pre-oxygenated and pre-medicated with $2 \mathrm{mg}$ midazolam and 100 mcg fentanyl. Then anesthesia was induced with Nesdonal at a dose sufficient to eliminate the eyelid reflex, and atracurium at a dose of $0.5 \mathrm{mg} /$ $\mathrm{kg}$ was used to facilitate intubation and muscle relaxation. We recorded the Nesdonal dose in the checklist.

To maintain anesthesia, $\mathrm{O}_{2}$ and $\mathrm{N}_{2} \mathrm{O}$ ratios of $50 \%$ and propofol with an appropriate dosage were used. If no anesthetic depth had been achieved despite the injection of propofol at a dose of $100 \mathrm{mcg} / \mathrm{kg} / \mathrm{min}$, then remifentanil was also used. The dose of propofol and remifentanil was also recorded in the checklist. 30 minutes before the end of surgery, methadone at a dose of $5 \mathrm{mg}$ was injected intramuscularly in all patients. At the end of the operation after complete awakening, the patient was given $40 \mathrm{mcg} / \mathrm{kg}$ neostigmine and $20 \mathrm{mcg} / \mathrm{kg}$ atropine for reversal of neuromuscular blockade and the tracheal tube was removed. Durations of all surgeries in the two groups were between 2.5 to 4 hours.

\section{Assessment of pain}

The severity of the pain was assessed by the Visual Analogue Scale (VAS) at 1, 2, 4, 6, 12 and 24 hours after patients entering the postanesthetic care unit (when the patient can express the intensity of pain according to the criteria). During the first postoperative 24 hours, vital signs were assessed at 1, 2, 4, 12 and 24 hours after the operation. In the case of $\operatorname{VAS}>4$, pethidine was administered intravenously at a dose of 0.5 $\mathrm{mg} / \mathrm{kg}$.

\section{Assessment of the relaxation rate}

After the finishing surgery, the patients' relaxation rate was assessed based on the Ramsay sedation score. The method of rating patients' relaxation was as follows: 1- patient awake, anxious, agitated or restless, 2- patient awake, cooperative, orientated and tranquil, 3patient drowsy with response to commands only, 4- patient asleep, brisk response to stimulus, 5patient asleep, sluggish response to stimulus, for example patient reacts with difficulty to a tap on the forehead or loud stimulus, 6- no response to firm nail-bed pressure or other noxious stimuli such as a tap on the forehead or loud stimulus. ${ }^{(20)}$

\section{Statistical analysis}

Data were analyzed by SPSS-23. Normality of data distribution was investigated using Kolmogorov Smirnov test. Comparison of qualitative variables or variables with non-normal distribution was performed using nonparametric statistical tests such as chi-square, Fisher exact test, and Mann-Whitney U test. Independent Samples t-test was used in the case of quantitative data with normal distribution. A p-value less than 0.05 was considered statistically significant. 


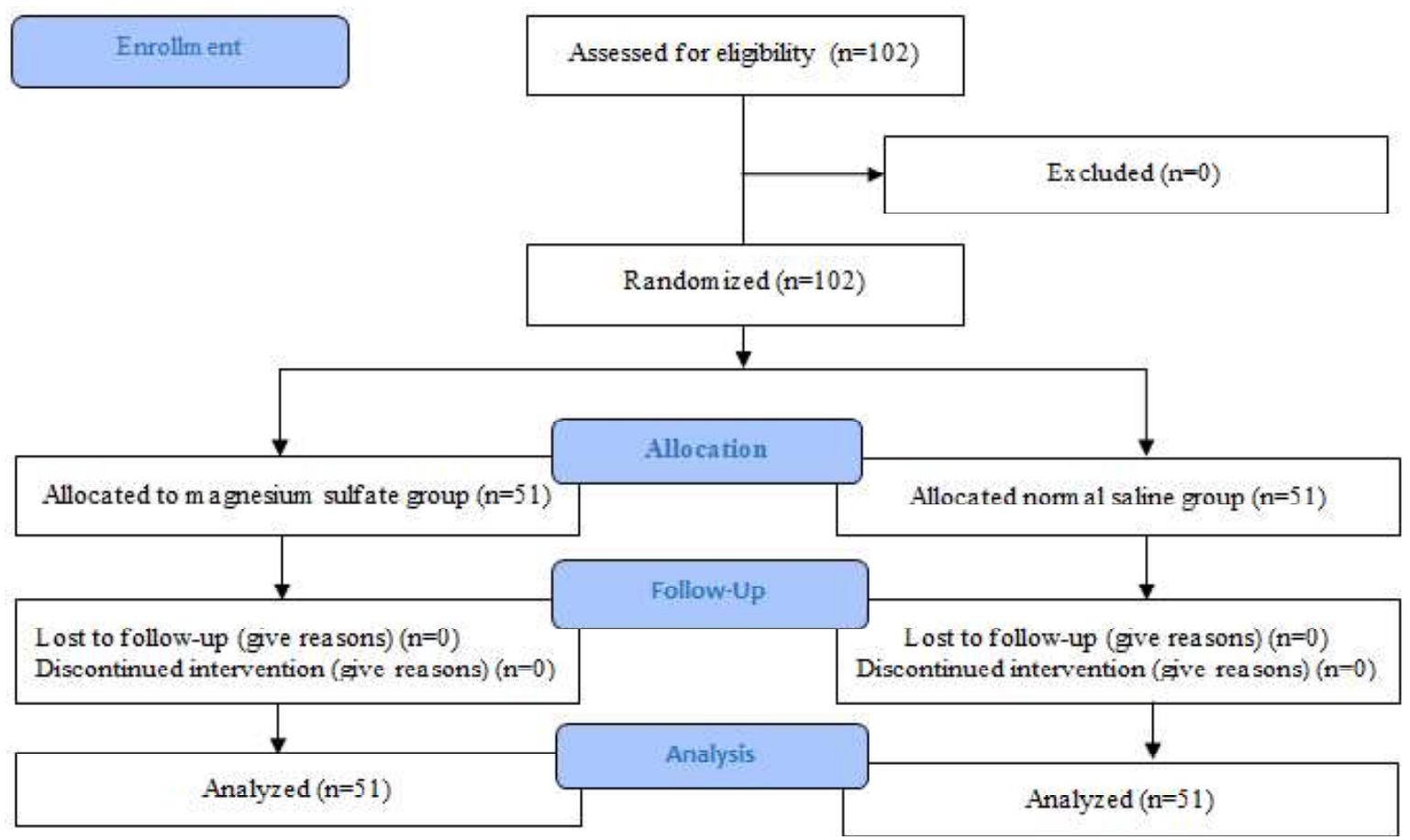

Figure 1. Flow diagram of the patients

\section{Ethical clearance}

The protocol of the study was approved by Ethical Committee of Baqiyatallah University of Medical Sciences under no. IR.bmsu.rec.1396. $604 \mathrm{cod}$. The informed consent form was received from each patient.

\section{RESULTS}

All 102 patients were divided into two groups, i.e. magnesium sulfate group $(n=51)$ and normal saline group $(n=51)$. All patients completed the trial (Figure 1).
At base-line there were no significant differences between the two groups regarding the age, sex and weight ( $\mathrm{p}=0.546, \mathrm{p}=0.529$ and $\mathrm{p}=0.086$ respectively). Also, there were no significant differences between the two groups regarding the heart rate, VAS, pethidine consumption, duration of surgery $(p=0.546$, $\mathrm{p}=0.186, \mathrm{p}=0.132$, and $\mathrm{p}=0.932$ respectively) (Table 1).

There was no significant difference at 2 , 4, 6, 12 and 24 hours post operation between two groups in the mean of VAS (pain). In the magnesium sulfate group $40(78.4 \%)$ and in the

Table 1. Comparison of demographic variables (age and sex), heart rate, blood pressure, VAS, pethedin consumption and duration of surgery between the two groups at base-line

\begin{tabular}{lccc}
\hline \multirow{2}{*}{ Characteristics } & \multicolumn{2}{c}{ Treatment groups } & \multirow{2}{*}{ p value } \\
\cline { 2 - 4 } & Magnesium (n=51) & Saline (n=51) & $0.546^{*}$ \\
\hline Age (years) & $53.25 \pm 13.87$ & $51.72 \pm 13.81$ & $0.529^{* *}$ \\
Sex (male/female) & $15 / 36$ & $19 / 32$ & $0.086^{*}$ \\
Weight (kg) & $78.50 \pm 7.78$ & $75.29 \pm 7.64$ & $0.546^{* * *}$ \\
Heart rate & $80.98 \pm 10.20$ & $79.79 \pm 9.51$ & $0.186^{* * *}$ \\
VAS $^{\dagger}$ & $4.96 \pm 1.35$ & $5.25 \pm 1.21$ & $0.132^{*}$ \\
Pethidine consumption (mg) & $23.72 \pm 9.78$ & $22.58 \pm 8.10$ & $0.932^{*}$ \\
Duration of surgery (minutes) & $194.54 \pm 120.21$ & $198.46 \pm 11.26$ &
\end{tabular}

Data presented as mean $\pm \mathrm{SD}$, except for gender; ${ }^{+V A S}$ : visual analog scale; ${ }^{*} \mathrm{~T}$-test, ${ }^{* *}$ Chi-square test, and ${ }^{* * * M a n n}$ Whitney test 
Table 2. Postoperative VAS scores at 2, 4, 6, 12 and 24 hours and the relaxation rate between the two groups

\begin{tabular}{cccc}
\hline & $\begin{array}{c}\text { Magnesium sulfate } \\
(\mathbf{n = 5 1 )}\end{array}$ & $\begin{array}{c}\text { Control } \\
(\mathbf{n = 5 1 )}\end{array}$ & p value \\
\hline VAS $^{\dagger}$ & $3.56 \pm 1.34$ & $3.82 \pm 1.12$ & $0.175^{*}$ \\
$2 \mathrm{~h}$ & $2.52 \pm 1.12$ & $2.41 \pm 0.96$ & $0.175^{*}$ \\
$4 \mathrm{~h}$ & $1.92 \pm 0.68$ & $1.90 \pm 0.75$ & $0.175^{*}$ \\
$6 \mathrm{~h}$ & $1.50 \pm 0.67$ & $1.68 \pm 0.70$ & $0.165^{*}$ \\
$12 \mathrm{~h}$ & $1.56 \pm 0.67$ & $1.35 \pm 0.56$ & $0.087^{*}$ \\
$24 \mathrm{~h}$ & & & \\
Relaxation rate $^{\S}$ & 6 & 8 & $0.393^{* *}$ \\
1 & 40 & 37 & \\
2 & 3 & 6 & \\
\hline & 2 & 0 & \\
\hline
\end{tabular}

Data presented as mean $\pm \mathrm{SD}$, except for relaxation rate. ${ }^{*} \mathrm{~T}$-test, and ${ }^{* *}$ Fisher exact test.; ${ }^{\dagger} \mathrm{VAS}$ : visual analog scale; \$1- patient awake, anxious, agitated or restless, 2- patient awake, cooperative, orientated and tranquil, 3patient drowsy with response to commands, 4- patient asleep, sluggish response to stimulus

control group $37(72.5 \%)$ patients were awake, cooperative, orientated and tranquil. There was no statistically significant difference between the two groups $(\mathrm{p}=0.393)$ (Table 2$)$.

There was no significant difference at 2 , 4 , and 6 hours post operation between the two groups in the mean of pethidine consumption (Table 3)

\section{DISCUSSION}

There was no significant difference between the two groups in pain and injection rate of pethidine. Finally, based on the RSS criteria, there was no significant difference in the level of relaxation between the two groups. Oguzhan et al. ${ }^{(17)}$ and Srivastava et al. ${ }^{(18)}$ demonstrated that magnesium sulfate was effective for postoperative opioid use and pain in patients following lumbar disc surgery and gynecological operation. But, in their study, they applied a continuous or repeat bolus in addition to the primary bolus of magnesium sulfate in opposition to single bolus preoperatively in the current research. It is essential to consider that there are some significant subjects associated with the studies performed on magnesium sulfate that could be the source of differences between their outcomes: various methods of anesthesia, techniques of "single dosage" versus "bolus plus infusion" dosage, and a complete dosage of intravenous magnesium sulfate. ${ }^{(9)}$ Our research has been carried out to assess the effectiveness and safety of preventive single-dose intravenous magnesium sulfate for postoperative pain reduction and analgesia. Furthermore, pain relief is multi-faceted and depends on culture,

Table 3. Comparison of pethidine consumption 2, 4, 6, 12 and 24 hours post operation between the two groups

\begin{tabular}{cccc}
\hline Pethidine consumption $(\mathbf{m g})$ & $\begin{array}{c}\text { Magnesium sulfate } \\
(\mathbf{n}=\mathbf{5 1})\end{array}$ & $\begin{array}{c}\text { Control } \\
(\mathbf{n}=\mathbf{5 1})\end{array}$ & p value \\
\hline $2 \mathrm{~h}$ & $11.76 \pm 12.60$ & $13.92 \pm 11.32$ & $0.441^{*}$ \\
4h & $2.15 \pm 6.72$ & $1.47 \pm 5.12$ & $0.688^{*}$ \\
$6 \mathrm{~h}$ & $0.21 \pm 1.90$ & $0.39 \pm 2.80$ & $0.317^{* *}$ \\
\hline
\end{tabular}

Data presented as mean $\pm \mathrm{SD}$. *T-test and **Mann-Whitney U test 
genetics, sex, race, socioeconomic state, cognition, and previous pain concept. Taheri et al. ${ }^{(10)}$ and Rezae et al. ${ }^{(4)}$ assessed the effect of single dose magnesium sulfate in patients with hysterectomy and cesarean section. They showed that pethidine consumption and postoperative pain were lower in the magnesium sulfate group. Pains following hysterectomy are multivariable and different from other pains e.g. surgery pain, visceral constructions pain, and dynamic pain (while straining, coughing and mobilizing). So, differences in the type of pains may be the sources of conflict between these studies. A meta-analysis showed that magnesium sulfate is capable of decreasing pain severity in early-stage and anesthetic consumption following laparoscopic cholecystectomy. ${ }^{(15)}$ A systematic review in patients undergoing orthopedic operation demonstrated that the studies did not give persuasive evidence of useful impacts on postoperative pain severity and the time to first analgesic demand. ${ }^{(16)}$ Due to the heterogeneity of orthopedic surgeries that were reported in their studies comparison of the duration of surgeries between the two studies ${ }^{(15,16)}$ is impossible. In another study Manaa et al. ${ }^{(9)}$ examined the effect of magnesium sulfate on postoperative anesthesia and analgesia in patients undergoing neurosurgical procedures. In this study, patients received magnesium sulfate (20 $\mathrm{mg} / \mathrm{kg}$ ) pre-operation then magnesium sulfate infusion $(10 \mathrm{mg} / \mathrm{kg} / \mathrm{h})$ continued until the end of surgery. Results showed that postoperative pain score, as well as a total analgesic requirement of morphine, was significantly lower in the magnesium group compared to the control group. Their results were in disagreement with the current study. Patients in magnesium group underwent bolus dose $20 \mathrm{mg} / \mathrm{kg}$ over five minutes before induction of anesthesia, continued through $10 \mathrm{mg} / \mathrm{kg} / \mathrm{h}$ as an infusion, in contrast to our study where the magnesium sulfate group received magnesium sulfate at a dose of $50 \mathrm{mg} / \mathrm{kg}$ in $20 \mathrm{~mL}$ volume and infused during 15-30 minutes pre-operation. A study by Elrahman et al. ${ }^{(21)}$ evaluated the role of preoperative magnesium sulfate guided transversus abdominis plane block in augmenting postcesarean analgesic effects. It started in the recovery unit where patients received lornoxicam, which is a non-steroidal substance. The mean pain at 6 and 12 hours postoperatively was significantly lower in the magnesium sulfate group than in the control group. The current study confirmed their results regarding the nonsignificant between-group differences in mean pain at other times. In the studies of Taheri et al. ${ }^{(10)}$ and Elrahman et al. ${ }^{(21)}$ the fact that analgesics were not used in both groups and/or non-steroidal analgesics were used to control postoperative pain was probably due to the potent effect of the opioid used. In the present study and studies consistent with the current study, the effectiveness of magnesium sulfate has been overshadowed by an opiate. So, differences in opioid consumption and pain in the two groups were not significant. However, in studies that did not use analgesics for postoperative pain control or where non-steroidal analgesics were used for analgesia, the effectiveness of magnesium sulfate was clearly seen in reducing pain and opioid consumption. However, in the studies of Rezae et al. ${ }^{(4)}$ and Manaa et al. ${ }^{(9)}$ who used opioids for postoperative analgesia, the difference between the two groups was significant. Kiran et al. ${ }^{(2)}$ reported that a single dose of intravenous magnesium sulfate could decrease post-operative pain in patients after inguinal operation. Also, the degree of relaxation was lower in patients in the group receiving magnesium sulfate than the control group. They also showed that patients receiving magnesium sulfate would be less likely to receive postoperative analgesics. Their results were in disagreement with the current study. It may be because of differences between type of patients and surgical conditions in the two groups.

Ghaffaripour et al. (22) investigated the effects of intravenous magnesium sulfate on postoperative analgesia after laminectomy. Magnesium sulfate was administered as a bolus followed by a maintenance dose up to the end 
of the surgery. They showed that the infusion of magnesium sulfate during laminectomy had no effect on patients' pain and opioid requirement postoperatively. Kahraman et al. ${ }^{(23)}$ showed that an infusion of magnesium sulfate has no effect on morphine consumption and postoperative pain in patients undergoing abdominal hysterectomy under spinal anesthesia. In the studies of Frassanito et al., ${ }^{(13)}$ Kahraman et al. ${ }^{(23)}$ and Ghaffaripour et al. ${ }^{(22)}$ whose results were consistent with the present study, morphine was used for postoperative analgesia, as we also used methadone for postoperative analgesia. Also, in the study of Frassanito et al. ${ }^{(13)}$ and Kahraman et al., ${ }^{(23)}$ patients were spinally anesthetized.

There were some limitations in our study. First, we applied only one dose of magnesium sulfate, while different regimens may have yielded different outcomes. Second, the infusion was restricted to the intraoperative interval only. Third, we did not evaluate the relationships between the magnesium level and the total anesthetic and analgesic demands. Finally, we did not assess the economical aspects of magnesium sulfate administration compared to other anesthetic medications. It is recommended to conduct more comprehensive studies to evaluate the effectiveness of magnesium sulfate in patients undergoing lumbar fusion surgery and in patients for whom the use of opiates is harmful (such as obese patients, etc.).

\section{CONCLUSION}

The results showed that pre-operative administration of magnesium sulfate has no effect on reduction of postoperative pain and opioid consumption in patients undergoing lumbar fusion surgery.

\section{CONFLICT OF INTEREST}

We declare that there is no conflict of interest in this study.

\section{ACKNOWLEDGEMENT}

We would like to thank the staff of the trauma research center, Baqiyatallah University of Medical Sciences for their kind cooperation in this research.

\section{CONTRIBUTORS}

All authors have contributed to the concept, design of methods, data gathering, data analysis and preparation of the final manuscript. All authors have read and approved the final manuscript.

\section{REFERENCES}

1. Dabbagh A, Elyasi H, Razavi S, et al. Intravenous magnesium sulfate for post-operative pain in patients undergoing lower limb orthopedic surgery. Acta Anaesthesiol Scand 2009;53:108891. doi: 10.1111/j.1399-6576.2009.02025.x.

2. Kiran S, Gupta R, Verma D. Evaluation of a singledose of intravenous magnesium sulphate for prevention of postoperative pain after inguinal surgery. Indian J Anaesth 2011;55:31-5. doi: 10.4103/0019-5049.76605.

3. Vadivelu N, Mitra S, Schermer E, et al. Preventive analgesia for postoperative pain control: a broader concept. Local Reg Anesth 2014;7:17-22. doi: 10.2147/LRA.S62160.

4. Rezae M, Naghibi K, Taefnia AM. Effect of preemptive magnesium sulfate infusion on the postoperative pain relief after elective cesarean section. Adv Biomed Res 2014;3:164. doi: 10.4103/ 2277-9175.139127.

5. Do SH. Magnesium: a versatile drug for anesthesiologists. Korean J Anesthesiol 2013;65:4. doi: 10.4097/kjae.2013.65.1.4.

6. Al Alawi AM, Majoni SW, Falhammar H. Magnesium and human health: perspectives and research directions. Int J Endocrinol 2018; 9041694. Doi: 10.1155/2018/9041694

7. Mishra AK, Afzal M, Mookerjee SS, et al. Preemptive analgesia: Recent trends and evidences. Indian J Pain 2013;27:114-20. doi: 10.4103/09705333.124582.

8. Yamanaka R, Shindo Y, Oka K. Magnesium is a key player in neuronal maturation and neuropathology. Int J Mol Sci 2019;20:3439. doi:10.3390/ijms20143439. 
9. Manaa E,Alhabib A. Effect of magnesium sulfate on the total anesthetic and analgesic requirements in neurosurgery. J Neurol Neurophysiol 2012; S11001. doi: 10:2155-9562.

10. Taheri A, Haryalchi K, Ghanaie M, et al. Effect of low-dose (single-dose) magnesium sulfate on postoperative analgesia in hysterectomy patients receiving balanced general anesthesia. Anesthesiol Res Pract 2015;2015:306145. doi: 10.1155/2015/306145.

11. Ryu JH, Kang MH, Park KS, et al. Effects of magnesium sulphate on intraoperative anaesthetic requirements and postoperative analgesia in gynaecology patients receiving total intravenous anaesthesia. $\mathrm{Br} \mathrm{J}$ Anaesth 2008;100:397-403. doi: 10.1093/bja/aem407.

12. Lysakowski C, Dumont L, Czarnetzki C, et al. Magnesium as an adjuvant to postoperative analgesia: a systematic review of randomized trials. Anesth Analg 2007;104:1532-9. doi: 10.1213/ 01.ane.0000261250.59984.cd.

13. Frassanito L, Messina A, Vergari A, et al. Intravenous infusion of magnesium sulfate and postoperative analgesia in total knee arthroplasty. Minerva Anestesiol 2015;81:1184-91.

14. Cavalcanti IL, de Lima FL, da Silva MJ, et al. Use profile of magnesium sulfate in anesthesia in Brazil. Front Pharmacol 2019;10:429. doi: 10.3389/ fphar.2019.00429.

15. Chen C, Tao R. The impact of magnesium sulfate on pain control after laparoscopic cholecystectomy: a meta-analysis of randomized controlled studies. Surg Laparosc Endosc Percutan Tech 2018;28:349-53. doi: 10.1097/ SLE.0000000000000571.

16. Peng YN, Sung FC, Huang ML, et al. The use of intravenous magnesium sulfate on postoperative analgesia in orthopedic surgery: a systematic review of randomized controlled trials. Medicine 2018;97:e13583. doi: 10.1097/MD.00000000000 13583.
17. Oguzhan N, Gunday I, Turan A. Effect of magnesium sulfate infusion on sevoflurane consumption, hemodynamics, and perioperative opioid consumption in lumbar disc surgery. J Opioid Manag 2008;4:105-10. doi: 10.5055/ jom.2008.0015.

18. Srivastava VK, Mishra A, Agrawal S, e al. Comparative evaluation of dexmedetomidine and magnesium sulphate on propofol consumption, haemodynamics and postoperative recovery in spine surgery: a prospective, randomized, placebo controlled, double-blind study. Adv Pharm Bull 2016;6:75-81. doi: 10.15171/ apb.2016.012.

19. Demiroglu M, Un C, Ornek DH, et al. The effect of systemic and regional use of magnesium sulfate on postoperative tramadol consumption in lumbar disc surgery. BioMed Res Int 2016, Article ID 3216246, 5 pages. http://dx.doi.org/10.1155/2016/ 3216246.

20. MottahedianTabrizi E, Tadrissi SD, Mohammad Yari A, et al. Validity and reliability of Ramsay sedation scale in Intensive Care Unit adult patients. Iranian J Critical Care Nursing Spring 2010;3;39-44.

21. Elrahman TNA, Youssry MA. The impact of single low dose IV magnesium sulphate adjuvant to ultrasound guided transverses abdominis plain block for control of postcesarean pain. Open J Obstet Gynecol 2017;7:269-80. DOI: 10.4236/ ojog.2017.73029.

22. Ghaffaripour S, Mahmoudi H, Eghbal H, et al. The effect of intravenous magnesium sulfate on post operative analgesia during laminectomy. Cureus 2016;8:e626 .doi: 10.7759/cureus.626.

23. Kahraman F, Eroglu A. The effect of intravenous magnesium sulfate infusion on sensory spinal block and post operative pain score in abdominal hysterectomy. Biomed Res Int 2014, Article ID 236024, 5 pages. http://dx.doi.org/10.1155/2014/ 236024. 